\title{
Encefalitis recurrente post vacuna influenza anual
}

\author{
Bernardita González y Alberto Fica
}

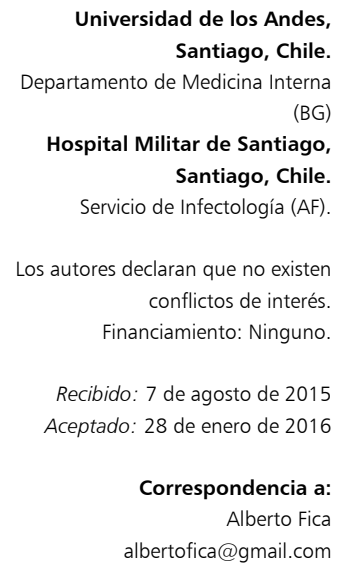

albertofica@gmail.com

\section{Recurrent encephalitis following annual influenza vaccine. Case report}

Influenza vaccine is rarely associated with neurological adverse effects such as Guillain Barré syndrome, encephalitis or aseptic meningitis. We report the case of a male patient that presented two episodes of acute encephalitis in consecutive years, 16 and 20 days after his annual influenza vaccine shot, respectively. In both instances, patient required ICU admission and evolved with fast recovery and no sequels. The first episode was managed empirically as herpetic encephalitis but an extensive study was performed in the second episode without identifying any microorganism. Neuroimaging studies also discarded acute disseminated encephalomyelitis. Mild pleocytosis of mononuclear predominance was detected in both cases in CSF. Naranjo score punctuated 8 points indicating a probable causal relationship. Acute encephalitis is a rare adverse effect of influenza vaccine and occurs several days after immunization. It has a broad differential diagnosis, and appears to be of self-limited duration and associated with good prognosis.

Key words: Influenza vaccine, adverse effects, encephalitis, central nervous system.

Palabras clave: Vacuna influenza, efectos adversos, encefalitis, sistema nervioso central.

\section{Introducción}

厂 a vacuna influenza es una herramienta fundamental de salud pública que ha permitido reducir morbilidad, ausentismo laboral o escolar, consultas médicas, gastos médicos, hospitalizaciones y muertes en diferentes grupos poblaciones ${ }^{1-12}$. En Chile, es administrada gratuitamente a diferentes grupos vulnerables y en el año 2015 se administraron más de 5 millones de dosis durante la campaña de vacunación.

Además de las molestias locales o fiebre, la vacuna administrada por vía parenteral puede rara vez generar efectos adversos neurológicos que incluyen encefalitis, meningitis aséptica, encefalomielitis aguda diseminada (EMAD), síndrome de Guillain Barré (SGB) o ataxia cerebelar aguda ${ }^{13-16}$. Se ha estimado a través de estudios de alta calidad, que la frecuencia del SGB alcanzó aproximadamente 1,6 casos adicionales por cada millón de dosis de vacuna monovalente A H1N1 ${ }^{17}$. Estudios post-comercialización durante una década (1994-2004) en Japón observaron las siguientes frecuencias de complicaciones neurológicas: nueve casos de SGB, tres casos de EMAD y un caso de ataxia cerebelar aguda, entre 38 millones de dosis aplicadas. No se observaron en este registro casos de encefalitis o meningitis aséptica secundarios a la vacuna ${ }^{13}$.

Comunicamos el caso clínico de un adulto mayor que presentó dos episodios de encefalitis aguda, luego de recibir la vacuna anual de influenza.

\section{Caso clínico}

Varón de 66 años con antecedentes de diabetes mellitus tipo 2 insulino-requiriente, hipertensión arterial, dislipidemia, gota, glaucoma bilateral y una meningo-encefalitis hacía un año que había sido tratada en otro hospital.

Consultó por un cuadro brusco de pocas horas de sensación febril y calofríos, con alteraciones conductuales (agresividad con familiares) y posteriormente rápido compromiso de conciencia. Una medición de glicemia capilar en domicilio descartó una hipoglicemia. Ingresó a un centro asistencial con un puntaje de la escala Glasgow de coma (GCS) de 14 puntos, confuso, desorientado y con agitación psicomotora (que se contuvo farmacológicamente con propofol, ketamina y lorazepam) y signos meníngeos negativos. Se realizó un estudio de imágenes con tomografía axial computarizada (TAC) y resonancia magnética (RM) de encéfalo que fueron informadas como normales. El análisis del LCR reveló un líquido incoloro, glucorraquia de $132 \mathrm{mg} / \mathrm{dL}$ (glicemia de 199 $\mathrm{mg} / \mathrm{dL}$ ), proteínas $249 \mathrm{mg} / \mathrm{dL}$, pleocitosis de 940 céls/ $\mathrm{mm}^{3}$ con $36 \%$ polimorfonucleares y $64 \%$ mononucleares, hematíes 100 céls $/ \mathrm{mm}^{3}, 100 \%$ frescos y tinción de Gram sin bacterias visibles. El estudio microbiológico del LCR con cultivo y reacción de polimerasa en cadena (RPC) para virus varicela zoster, virus herpes simplex 1,2 y 6 , citomegalovirus, test de látex para $N$. meningitidis, hemocultivos y urocultivo, fueron todos negativos. En los exámenes generales destacaba una leucocitosis 
de 14.850 céls $/ \mathrm{mm}^{3}$, neutrofilia (82\%), velocidad de eritrosedimentación (VHS) discretamente elevada (21 $\mathrm{mm} / \mathrm{h}$ ), pruebas bioquímicas y hepáticas normales, salvo una hiperglicemia y alza leve de la creatinina plasmática $(1,3 \mathrm{mg} / \mathrm{dL})$. Los valores de proteína $\mathrm{C}$ reactiva fueron normales $(5,5 \mathrm{mg} / \mathrm{L})$ y de procalcitonina bajos $(<0,05 \mathrm{ng} /$ $\mathrm{mL})$. Evolucionó con una hipotensión arterial $(96 / 56 \mathrm{~mm}$ $\mathrm{Hg}$ ) que respondió a la administración de volumen, además de compromiso de conciencia fluctuante (Glasgow bajó a 11). Se inició terapia antimicrobiana empírica con ceftriaxona, ampicilina y aciclovir i.v. y glucocorticoides (dexametasona $8 \mathrm{mg}$ ). Se trasladó a nuestro hospital donde ingresó en Glasgow fluctuante (entre 10 y 13 puntos), con hemodinamia estable, febril $\left(38,5^{\circ} \mathrm{C}\right)$, con reflejos osteotendíneos exacerbados, sin focalidad neurológica, signos meníngeos o lesiones embólicas sistémicas o cutáneas. Se repitió el estudio de LCR, en cuyo citoquímico las proteínas disminuyeron a $81 \mathrm{mg} / \mathrm{dL}$ y la pleocitosis a 40 céls $/ \mathrm{mm}^{3}$. El estudio en LCR para tuberculosis (adenosina deaminasa, baciloscopia y cultivo de Koch), enterovirus (RPC) y hongos (tinta china y cultivo) fueron negativos y/o normales. Se descartó también una neumonía por imágenes y la serología para VIH fue negativa.

El paciente evolucionó favorablemente, se logró desconectar del ventilador mecánico a las $24 \mathrm{~h}$ de su ingreso, sin demostrar focalización neurológica alguna y recuperando totalmente sus funciones cerebrales superiores. Los antimicrobianos fueron suspendidos a las $72 \mathrm{~h}$. $\mathrm{Al}$ interrogatorio dirigido, el paciente no refirió ningún síntoma respiratorio alto o bajo (rinorrea, tos, odinofagia o disfonía) que justificara la búsqueda de virus respiratorios. Fue dado de alta al cuarto día de su ingreso.

Se obtuvieron antecedentes de la meningoencefalitis ocurrida un año atrás, según la epicrisis. Había estado internado 12 días, dos de ellos en ventilación mecánica. El estudio del LCR indicó ausencia de bacterias a la tinción de Gram, pleocitosis de 70 céls $/ \mathrm{mm}^{3}$ ( $60 \%$ mononuclear), proteínas de $68 \mathrm{mg} / \mathrm{dL}$ y glucosa de $135 \mathrm{mg} / \mathrm{dL}$. El cuadro se interpretó como viral, tratándose con aciclovir i.v. por 10 días, sin realizarse estudio viral del LCR.

En forma dirigida se rescató el antecedente de vacunación anual contra la influenza (vacuna trivalente) en los últimos cuatro años consecutivos (2011 al 2015). En el año 2014, la vacuna había sido administrada 16 días antes del cuadro de meningoencefalitis (Vaxigrip ${ }^{\circledR}$, Sanofi, lote K7375-2) y en el último episodio, producido en el año 2015, 20 días antes (Influval ${ }^{\circledR}$, Abbott, lote HY01N). El análisis de causalidad, según la puntuación de Naranjo dio 8 puntos $^{18}$.

\section{Discusión}

Los eventos de encefalitis en el caso presentado quedan, a nuestro juicio, respaldados por un cuadro neurológico agudo con fiebre, compromiso de conciencia y pleocitosis en el LCR. A su vez, el rol de la vacuna influenza queda sugerido por el carácter recurrente del mismo cuadro clínico con aproximadamente la misma latencia y la ausencia de una etiología infecciosa específica luego de un amplio estudio, al menos en el segundo episodio. La posibilidad de EMAD fue descartada por la ausencia de lesiones en la sustancia blanca en el estudio de $\mathrm{RM}^{13,19}$. El análisis de causalidad, usando los criterios de Naranjo, sumó 8 puntos, estableciendo una relación probable (definitiva si $\geq 9$ puntos) ${ }^{18}$. Creemos que causas alternativas para este cuadro fueron descartadas por los estudios efectuados, tanto microbiológicos, serológicos y de imágenes y por la rápida recuperación neurológica, sin secuelas.

Los criterios de Naranjo fueron publicados en los años 80 y permitieron sistematizar los efectos adversos asociados a terapias médicas, disminuyendo la variabilidad entre los observadores, ofreciendo al mismo tiempo una estimación de la posible relación causal entre los síntomas y el fármaco o compuesto comprometido. Consiste en la aplicación de un cuestionario de 10 preguntas, con un puntaje asignado a las respuestas posibles. Notablemente las preguntas de mayor puntaje corresponden a la asociación temporal y a la reproducción de los síntomas frente a una segunda exposición ${ }^{18}$.

Como se anticipó en la introducción, los eventos de encefalitis post-vacuna influenza son extraordinariamente infrecuentes y escasamente reportados en la literatura médica. Los casos descritos suelen presentarse entre una y dos semanas después de la administración de la vacuna. Se asocian a un compromiso de conciencia transitorio, pleocitosis mononuclear, sin hipoglucorraquia o proteinorraquia importante y tienen en la mayor parte de los casos un desenlace favorable sin secuelas como en nuestro paciente, incluso luego de dos eventos ${ }^{15}$. Los escasos casos publicados no parecen haber recibido terapia corticoesteroidal o inmunosupresora, a diferencia de nuestro caso ${ }^{15}$. No tenemos conocimiento de reportes de encefalitis recurrentes luego de cada vacuna anual, lo que hace que este caso sea excepcional. Para evitar nuevos eventos, se le recomendó al paciente no recibir nuevas dosis de vacuna influenza.

En conclusión, los episodios de encefalitis post-vacuna influenza son extremadamente infrecuentes y ocurren pocas semanas después de la administración de la vacuna. Requieren un amplio diagnóstico diferencial, son autolimitados y se asocian a un buen pronóstico.

\section{Resumen}

La vacuna influenza en raras ocasiones se asocia a efectos adversos neurológicos tales como síndrome de 
Guillain Barré, encefalitis o meningitis aséptica, entre otros. Presentamos el caso clínico de un paciente de sexo masculino de 66 años que presentó dos episodios de encefalitis en años consecutivos, 16 y 20 días después de recibir la vacuna anual de influenza, respectivamente. En ambos casos requirió ingreso a UCI y evolucionó con una rápida recuperación y sin secuelas. El primer episodio fue manejado empíricamente como una encefalitis herpética y en el segundo se hizo un extenso estudio microbioló- gico que no identificó microorganismos. Los estudios de neuroimágenes descartaron una encefalomielitis aguda diseminada. En ambos casos se pesquisó pleocitosis de predominio mononuclear en el LCR. El score de Naranjo dio 8 puntos indicando una probable relación causal. La encefalitis aguda es un efecto adverso muy infrecuente tras la vacuna influenza y ocurre varios días después de la inmunización. Tiene un amplio diagnóstico diferencial, parece ser autolimitado y de buen pronóstico.

\section{Referencias bibliográficas}

1.- $\quad$ Beyer W E, McElhaney J, Smith D J, Monto A S, Nguyen-Van-Tam J S, Osterhaus A D. Cochrane re-arranged: support for policies to vaccinate elderly people against influenza. Vaccine 2013; 31: 6030-3.

2.- Nichol K L, Goodman M. The health and economic benefits of influenza vaccination for healthy and at-risk persons aged 65 to 74 years. Pharmacoeconomics 1999; 16 Suppl 1: 63-71.

3.- Osterholm M T, Kelley N S, Sommer A, Belongia E A. Efficacy and effectiveness of influenza vaccines: a systematic review and meta-analysis. Lancet Infect Dis 2012; 12: 36-44.

4.- Norhayati M N, Ho J J, Azman M Y. Influenza vaccines for preventing acute otitis media in infants and children. Cochrane Database Syst Rev 2015; 3: CD010089.

5.- Remschmidt C, Wichmann O, Harder T. Vaccines for the prevention of seasonal influenza in patients with diabetes: systematic review and meta-analysis. BMC Med 2015; 13: 53 .

6.- Remschmidt C, Wichmann O, Harder T. Influenza vaccination in patients with end-stage renal disease: systematic review and assessment of quality of evidence related to vaccine efficacy, effectiveness, and safety. BMC Med 2014; 12: 244.

7.- Remschmidt C, Wichmann O, Harder T. Influenza vaccination in HIV-infected individuals: systematic review and assessment of quality of evidence related to vaccine efficacy, effectiveness and safety. Vaccine 2014; 32: 5585-92.

8.- Eliakim-Raz N, Vinograd I, Zalmanovici Trestioreanu A, Leibovici L, Paul M. Influenza vaccines in immunosuppressed adults with cancer. Cochrane Database Syst Rev 2013; 10 : CD008983.

9.- Zaman K, Roy E, Arifeen S E, Rahman M, Raqib M, Wilson E, et al. Effectiveness of maternal influenza immunization in mothers and infants. N Engl J Med 2008; 359: 1555-64.

10.- Madhi S A, Cutland C L, Kuwanda L, Weinberg A, Hugo A, Jones S, et al. Influenza vaccination of pregnant women and protection of their infants. N Engl J Med 2014; 371 : 918-31.

11.- Lemaitre M, Meret T, Rothan-Tondeur M, Belmin J, Lejonc J L, Luquel L, et al. Effect of influenza vaccination of nursing home staff on mortality of residents: a cluster-randomized trial. J Am Geriatr Soc 2009; 57: 1580-6.

12.- Potter J, Stott D J, Roberts M A, Elder A G, O'Donnell B, Knight P V, et al. Influenza vaccination of health care workers in long-termcare hospitals reduces the mortality of elderly patients. J Infect Dis 1997; 175: 1-6.

13.- Nakayama T, Onoda K. Vaccine adverse events reported in post-marketing study of the Kitasato Institute from 1994 to 2004. Vaccine 2007; 25: 570-6.

14.- Yahr M D, Lobo-Antunes J. Relapsing encephalomyelitis following the use of influenza vaccine. Arch Neurol 1972; 27: 182-3.

15.- Rosenberg G A. Meningoencephalitis following an infuenza vaccination. N Engl J Med 1970; 283: 1209.

16.- Wells $C \mathrm{E}$. A neurological note on vaccination against influenza. Br Med J 1971; 3: 755-6.

17.- Agency for Healthcare Research and Quality. Safety of vaccines used for routine immunization in the United States. Executive Summary. Evidence Report/Technology Assesment number 215. Disponible en: http:// www.ahrq.gov/research/findings/evidencebased-reports/vaccinestp.html. (Fecha acceso: julio de 2015).

18.- Naranjo C A, Busto U, Sellers E M, Sandor P, Ruiz I, Roberts E A, et al. A method for estimating the probability of adverse drug reactions. Clin Pharmacol Ther 1981; 30: 239-45.

19.- Olivares F, Salinas M, Soto A, Dabanch J, Fica A. Encefalomielitis aguda diseminada grave. Reporte de un caso asociado a infección por virus parainfluenza 3. Rev Chilena Infectol 2015; 32: 476-81. 